\title{
Proteomics in Gerontology: Current Applications and Future Aspects - A Mini-Review
}

\author{
E. Schiffer ${ }^{\mathrm{a}}$ H. Mischak ${ }^{\mathrm{a}}$ L.U. Zimmerli ${ }^{\mathrm{b}}$

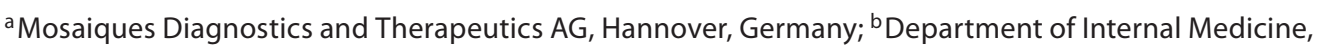 \\ University Hospital Zürich, Zürich, Switzerland
}

\section{Key Words}

Coronary artery disease - Diabetic nephropathy ·

Neoplasia $\cdot$ Proteomics that allow comparison of datasets provided by different scientists. Clearly, the proteome is by far too complex to be tackled by one laboratory on its own.

Copyright $\odot 2009$ S. Karger AG, Basel

\begin{abstract}
Background: Aging is closely related to the onset of chronic diseases, such as coronary artery disease, diabetic nephropathy or different types of malignancies, reflecting the demand for novel biomarkers to manage theses diseases. $\mathbf{O b}$ jective: The analysis of the human proteome for biomarkers has made considerable advances in the last years. Methods: We describe the main technological approaches taken, their advantages and disadvantages. Results: We will review the different clinical sources of material and attempt to highlight the different challenges and approaches associated with these. Age-related changes in the proteome have been described and were found to be highly similar to changes associated with chronic diseases. We will give several examples on the successful application of proteomics in the diagnosis, prognosis and therapy of these chronic diseases. Conclusions: A boost in disease-related proteomic information is expected in the very near future, and will also result in its broad clinical application. However, this view appears to be dependent on the strict adherence to proper technological/ analytical parameters, correct statistics, and large databases
\end{abstract}

\section{Introduction}

With age, the prevalence of death due to chronic diseases increases steeply. Most deaths are due to cardiovascular diseases, with a prevalence of up to $50 \%$ in the growing elderly population [Euro Heart Survey, Cardiovascular Diseases in Europe 2006; http://www.escardio. org/guidelines-surveys/ehs/Documents/EHS-CVD-report-2006.pdf]. The burden of cost related to these diseases will increasingly challenge public health systems. Therefore, diagnostic biomarkers have a great potential for the early diagnosis, monitoring and prognosis of a variety of chronic diseases.

Classical approaches for biomarker discovery are hypothesis-driven, thus related to extensive biochemical research to understand pathological processes and to indentify possible candidate markers. In contrast, technical advances over the last decades, especially in mass spectrometry (MS) and computation, have allowed a paradigm shift into the direction of multi-parametric 'omic'

\section{KARGER}

Fax +4161306 1234

E-Mail karger@karger.ch

www.karger.com (c) 2009 S. Karger AG, Basel

0304-324X/09/0552-0123\$26.00/0

Accessible online at:

www.karger.com/ger
Eric Schiffer

Mosaiques Diagnostics and Therapeutics AG

Mellendorfer Strasse 7-9, DE-30625 Hannover (Germany)

Tel. +49511 554744 17, Fax +49511 55474431

E-Mail schiffer@mosaiques-diagnostics.com 
approaches for biomarker discovery. All classes of biological compounds, from genes, over mRNA to proteins and metabolites, can be analyzed by the respective omic approaches, namely genomics, transcriptomics, proteomics or metabolomics. The number of genes is around 20,000 compared to $1,000,000$ estimated proteins and roughly 5,000 metabolites [1]. While the data obtained from genomics may explain the disposition of diseases, e.g., increased risk of acquiring a certain disease, several other environmental, not gene-mediated mechanisms may be involved in the onset of diseases. Furthermore, a single gene can be processed to result in several different mRNAs or proteins, which directly determine different cellular functions. Changes in metabolite fluxes, as a downstream result of gene expression and protein translation, are expected to be amplified relative to changes in the transcriptome and proteome. However, these fluxes vary quickly and imply that time-dependent measurements and considerations of metabolite content only at a certain time point can be misleading [2]. It may be appropriate to conclude that genomics enables assessing all potential information, proteomics enables assessing the actually executed programs, and metabolomics will mostly display the results of such execution. Therefore, a broader view of the pathology of diseases is likely to be achieved using a proteomic approach.

The analysis of the human proteome has made considerable advances in the last years and is prepared to overcome several obstacles for its routine application. Of utmost consideration is the source of material to be used. We will therefore review the different sources of samples used for clinically oriented analysis, and attempt to highlight the different challenges and approaches associated with these. Additionally, we want to shortly describe the main technological approaches taken, their advantages and disadvantages.

Given the high biological variability as a serious methodological impediment, it appears imperative to evaluate clinical conditions not on the basis of single peptide markers, but rather on the basis of a panel of biomarkers derived from distinct and clearly defined molecules. A panel of biomarkers will tolerate changes in individual analytes without jeopardizing the diagnostic precision, i.e., such variability will not result in gross changes of the diagnostic result.

A major challenge of proteome analysis - as of most other multiple parametric tests - is the statistical evaluation of datasets and combinations of single markers to specific biomarker models. However, the high number of variables also implies an enormous potential benefit: multiple (patho-)physiological conditions can be assessed based on a single analysis, provided the indicative biomarkers for these different conditions are known and can be analyzed with sufficient accuracy.

We give several examples on the successful application of proteome analysis in the detection of chronic diseases, such as diabetes, diabetic nephropathy, coronary artery disease (CAD), or highly prevalent malignancies. In addition, evaluation of therapeutic approaches and prognosis of disease progression have recently been demonstrated.

\section{Biological Samples}

For clinical proteomics, the careful selection of sample material is of utmost importance. In principle, tissues and body fluids and also cell cultures can be analyzed. Certainly, tissue samples are closely associated with pathological changes, and improvements in the field of laser capture microdissection allow sampling of homogenous tissue cell populations, but tissue sampling is always related to invasive procedures. In contrast, the access to many body fluids is minimally or non-invasive.

In general, polypeptides of biological samples are exposed to proteolytic activity and are therefore prone to degrade. For example, a detailed evaluation of the analytical stability of blood serum revealed high proteolytic activity activated immediately upon clotting, resulting in fast degradation and generation of proteolytic products. Consequently, the Human Proteome Consortium (HUPO) recommends analyzing blood in the form of plasma rather than serum and has established a standardized sample collection protocol [3]. Although proteolytic activity in blood plasma is low compared to blood serum, considerable residual activity endangering reliable proteome analysis can be observed [Muhlen et al., manuscript in press]. In contrast, urinary proteins were demonstrated to be stable enough for reliable proteome analysis $[4,5]$. In this context, the systematic work of Fiedler et al. [6] demonstrated the various influences of endogenous and exogenous variables on urine peptide patterns, such as urine sampling conditions, urine storage, freeze-thaw cycles and blood/bacterial interferences. To minimize these biases, standardized protocols for urine sampling and handling are proposed [7] and should be mandatory for other types of biological samples, especially as those are of generally much larger variability than urine. 
A crude unprocessed biological sample can be considered an ideal source for a proteomic profiling experiment avoiding any artifact or bias arising from sample preparation. Unfortunately, the presence of interfering compounds, such as aggregates, lipids, carbohydrates, etc., often prevent such an approach or let it often appear as impractical. Consequently, sample preparation protocols should be tested for minimal or at least reproducible loss of polypeptides. Reproducibility of sample preparation is one of the most important considerations and cannot be overemphasized. Each additional step during preparation introduces additional artifacts to the complex system. Hence, it appears advisable to remove biological matrix and other low-molecular weight compounds in a single step.

\section{Technical Considerations}

Even exactly defined proteomes are highly complex mixtures preventing their analysis in a single MS step. Therefore, an additional separation step before applying MS analysis (pre-MS separation) has to be introduced to increase overall analytical resolution. Here, conventional separation techniques, such as liquid chromatography (LC) or capillary electrophoresis (CE), are followed by subsequent MS analysis. A detailed overview into the different ionization processes and into modern mass spectrometers would be far beyond the scope of this review [for more detailed information, see 8, 9]. Briefly, ionization of polypeptides as a whole (without its disassembling) in a so-called soft ionization process can technically be achieved using either matrix-assisted laser desorption ionization (MALDI) or electrospray ionization (ESI).

For MALDI, the sample is mixed with a matrix and spotted onto a target plate for co-crystallization. A pulsed laser beam desorbs analytes, whereas the matrix molecules absorb the energy of the laser and transfer it to the analyte. For proteomic applications, an entire LC or CE separation run is spotted offline (without physical connection to the MS) onto the target plate, and subsequently MS analysis is performed. Offline coupling is technically less demanding, but tends to 'signal suppression', an effect observed when certain analytes are preferentially ionized and hence are easily detected, but at the expense of other analytes that may even become undetectable [10].

Pre-MS separation can also be coupled online (with physical connection) to MS devices as is generally done with ESI, which generates charged droplets in a highvoltage field. In this field, droplet solvent progressively evaporates, generating multiply charged analyte ions. This approach may be less stable compared to MALDI due to possible electrospray collapses, but is less susceptible to signal suppression effects.

Besides profiling of biological samples, determination of peptide sequences of candidate biomarkers is possible as well by using MS approaches (fig. 1). Here, two MS-devices are physically connected to each other (therefore tandem MS or MS/MS). The first MS filters or isolates the ions of interest (so-called 'parent ions'). In a second step, the isolated parent ions are subjected to fragmentation by either collision with other molecules (collision-induced dissociation) or by the transfer of electrons to the target molecule (electron transfer dissociation). The obtained fragments (so-called 'daughter ions') are analyzed using the second MS instrument (fig. 1). The obtained MS/MS spectrum of the fragments displays the sequence of the parent ion, which is routinely searched against databases to identify the protein from which the biomarker originated.

\section{Proteomics Platforms}

Over the last decades, mainly five different proteomic technology platforms were applied, namely two-dimensional gel electrophoresis (2DE), surface-enhanced laser desorption/ionization (SELDI), LC-MS, CE coupled to MS (CE-MS), and protein arrays (fig. 2). In the following section, the different platforms will be introduced and their advantages and disadvantages will be discussed briefly.

2DE separates proteins $(>10 \mathrm{kDa})$ according to their isoelectric point and molecular mass (Da). Protein identification is routinely performed in a step-by-step approach based on proteolytic in-gel digest, followed by gel extraction, and MS analysis of the resultant peptide fragments [11]. In order to reduce gel-to-gel variability and to increase the reliability of protein quantification, two-dimensional difference gel electrophoresis (2D-DIGE) was developed. The method utilizes two samples differentially labeled with fluorescent dyes (e.g. Cy3 and Cy5). Subsequently, the two samples are resolved simultaneously within the same gel. While the approach allows satisfactory comparison of two samples [12], the comparison of several different experiments remains challenging. In summary, 2DE provides high resolution protein separation and direct information on expression levels of intact 


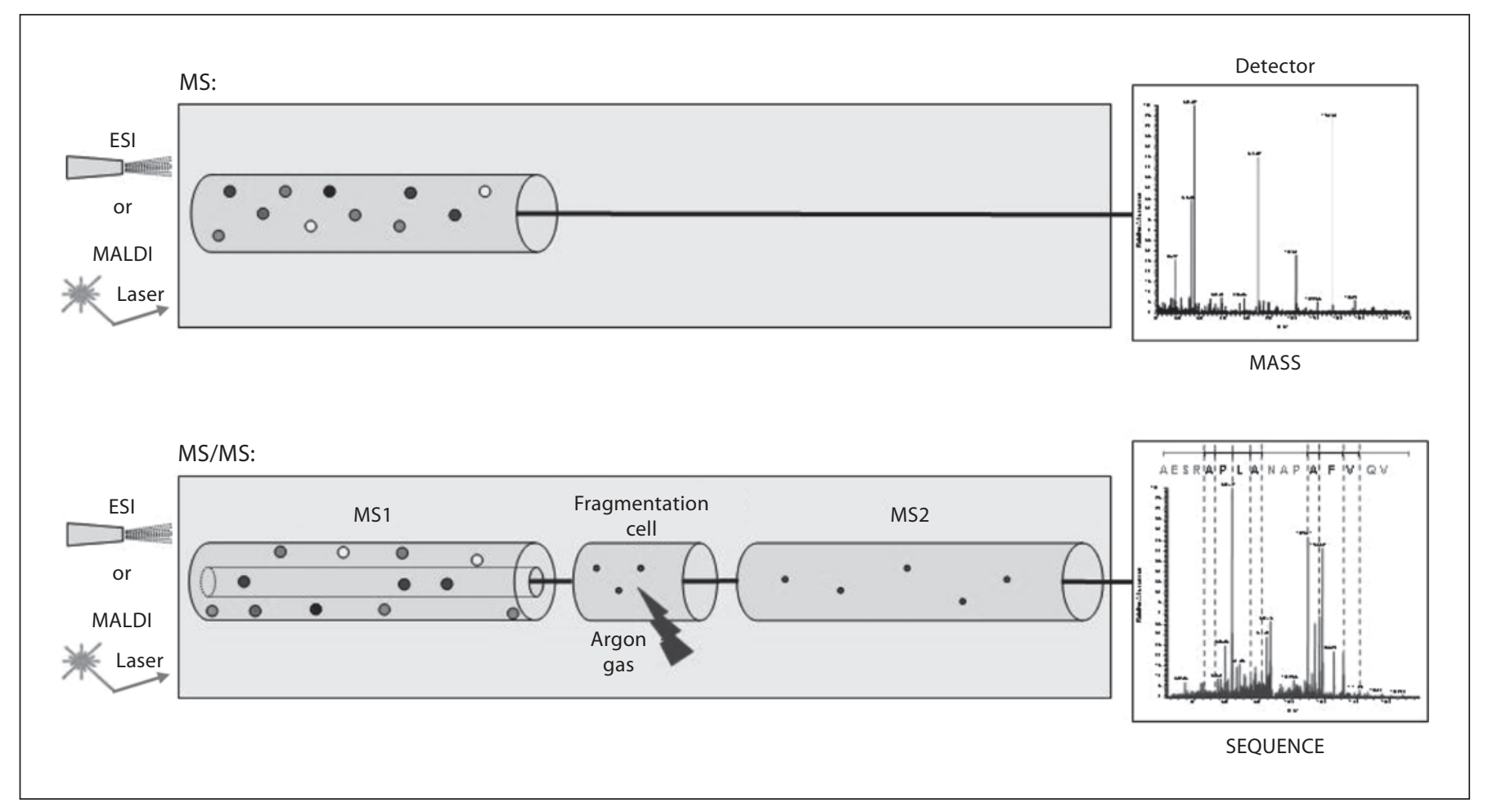

Fig. 1. Tandem mass spectrometry for peptide sequencing. Mass spectrometric (MS) approaches are suitable to determine the peptide sequences of candidate biomarkers. The upper panel schematically depicts classical MS analysis of analytes. Ionization is either achieved by electrospray ionization (ESI) or matrix-assisted laser desorption/ionization (MALDI). The mass of analytes is determined and displayed in the mass spectrum (upper right panel). For peptide sequencing, two MS devices are physically connected to each other (lower panel). The first MS filters or isolates the ions

proteins. Methodologically, 2DE is rather time-consuming, due to a certain extent to the lack of automation, and inter-assay comparability is difficult to achieve [13]. However, 2DE still appears to be the method of choice for comparative analysis of large proteins.

SELDI attempted to resolve several limitations of 2DEMS and was applied in a number of clinically relevant proteome investigations. SELDI utilizes selective interactions of polypeptides with different chip surfaces to achieve fractionation of biological samples and thereby to reduce their complexity for subsequent MALDI-MS analysis. Numerous matrices serve as 'active' surfaces, such as hydrophilic material, reversed-phase material, or affinity reagents, such as lectins or antibodies. The aim is to bind a small, but well-defined fraction to the surface, depending on the concentration, $\mathrm{pH}$, salt content, presence of interfering compounds like lipids, etc. Unfortunately, ex- of interest (so-called 'parent ions'). In a second step, the isolated parent ions are subjected to fragmentation by either collision with other molecules (collision-induced dissociation) or by the transfer of electrons to the target molecule (electron transfer dissociation). The obtained fragments (so-called 'daughter ions') are analyzed using the second MS instrument. The obtained MS/MS spectrum of the fragments (lower right panel) displays sequence of the parent ion, which is routinely searched against databases to identify the protein from which the biomarker originated.

actly this core element represents SELDI's major analytical obstacle: reproducibility of binding appears to be low and the inability to detect low abundance peptides and proteins - in part due to the limited resolution of the applied mass spectrometer - as well as the inability to directly identify discriminatory peaks are major drawbacks. Currently, efforts are underway to alleviate at least some of the reproducibility issues by strict technical control [14], by the use of higher resolution mass spectrometers, and alternative sample preparation and pre-fractionation strategies [15]. Recently, material-enhanced laser desorption/ionization (MELDI) was introduced to handle the low reproducibility of binding to SELDI surfaces by utilizing various functionalized porous carrier materials with enlarged active binding surfaces [16].

If analytical sensitivity is being considered, LC-MS provides high-resolution separation with a high capacity 


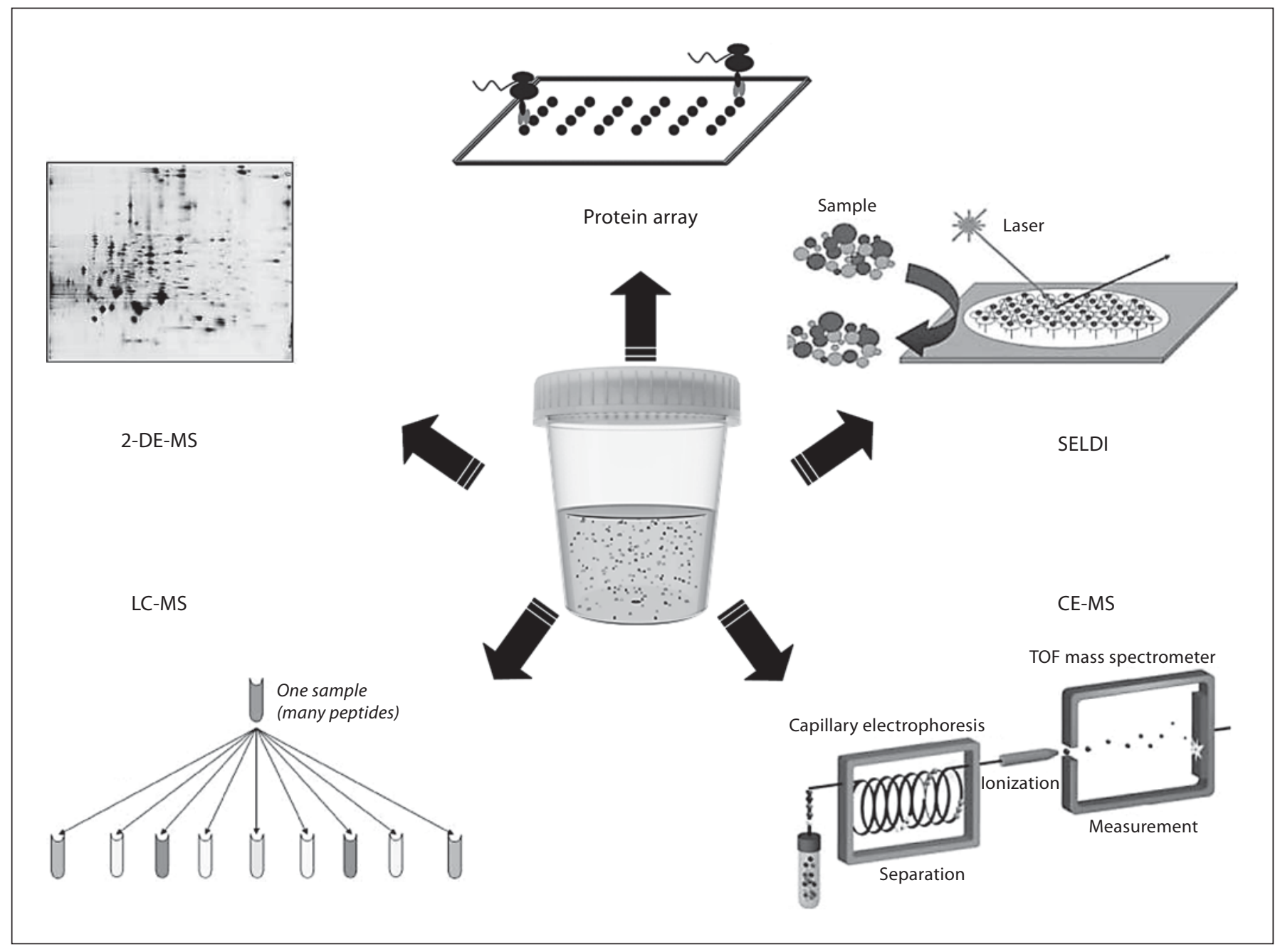

Fig. 2. Proteomics platforms. Mainly five different proteomic technology platforms are routinely applied: two-dimensional gel electrophoresis (2DE), surface-enhanced laser desorption/ionization (SELDI), liquid chromatography mass spectrometry (LCMS), capillary electrophoresis coupled to mass spectrometry (CEMS), and protein arrays. 2DE separates proteins $(>10 \mathrm{kDa})$ according to their isoelectric point (pI) and molecular mass (Da).

for analytes that can be loaded onto an LC column [for a more in-depth review, see 17]. To deepen analysis, sequential separation with different matrices can be performed in two independent steps. Approaches like multidimensional protein identification technology [18] and 2D liquid phase fractionation [19] provide immense amounts of data. Generally, LC-MS tends to be time-consuming, hence limiting its application in routine clinical analysis. Additionally, $\mathrm{LC}$ is sensitive towards interfering compounds and precipitation of analytes on column materials [13], complicating separation of large analytes cov-
SELDI utilizes selective interactions of polypeptides with different chip surfaces to achieve fractionation of biological samples. LC-MS provides high-resolution separation with a high capacity for analytes. CE-MS is relatively robust and compatible with almost all kinds of volatile buffers. Protein microarrays detect specific proteins in a non-MS-based 'targeted proteomics' approach. ering broad ranges of hydrophobicity. In the last decade, monoliths were applied in mircoscale chromatographic separation, such as $\mu$-LC-MS [20], to tackle these limitations due to easy column preparation, fritless design, high permeability, low backpressure, fast analyte mass transfer, and versatile surface chemistry [21].

In contrast, CE-MS used free-flow separation of analytes in buffer-filled capillaries [22]. Therefore, CE-MS is relatively robust and compatible with almost all kinds of volatile buffers without continuous adjustment of the ionization voltage for optimal ESI [23], due to the absence 
of buffer gradients, as is the case in LC-coupled MS analysis. Therefore, CE-MS certainly appears to be an excellent choice for the fast high resolution analysis of complex biological samples. However, CE-MS is not well suited for the analysis of proteins of $>20-30 \mathrm{kDa}$ due to at least partial precipitation of these proteins when acidic running buffers are used, as is frequently the case. The problem can be addressed by depletion of those analytes using ultrafiltration [24].

In contrast to the already discussed platforms, protein microarrays detect specific proteins in a non-MS-based 'targeted proteomics' approach. The technique prints specific antibodies or antigens on a slide or a membrane to facilitate immune detection of multiple proteins. A single sample is hybridized to the array followed by the detection of the captured antigens or antibodies [25]. However, not only the demand for target peptide sequence information and a highly specific probe for every target molecule (in contrast to nucleic acid arrays, no anti-sense sequence can be used) restricts broad applicability, but the low density also limits detection to a small set of proteins without addressing post-translational modifications [25].

\section{Processing of Proteomic Data}

Proteomic biomarker discovery can be considered a sophisticated search for a needle in a haystack. The immense data of a single proteomic analysis require adequate tools for data analysis. The essentials to be extracted are identity and quantity of detected polypeptides. Therefore, protein quantification is of essential relevance. For gels, protein stains, such as Coomassie blue or silver stains, are routinely used for quantification. More recently, fluorescent dyes provide larger linear dynamic ranges in combination with similar or better sensitivity [26]. For MS-based proteomics, relative quantification based on ion counting is available, demanding care in instrument and technical consistency [27]. Absolute quantification is usually achieved using an isotope-labeled marker analog. The need for prior successful identification of a target sequence and/or its chemical derivatization is certainly restrictive. Generally, relative quantification with reference to constant peaks appears sufficient, especially considering given biological variation [Jantos-Siwy et al., manuscript in press].

For comparative data evaluation, it is essential to be able to identify identical polypeptides with high probability in consecutive samples. Hence, the resolution and accuracy of the parameters used for target definition are of major importance, such as retention time for LC separation, migration time for CE separation, and molecular mass. Separation-based parameters often vary with the ion content of the samples, pinpointing the need for thorough data normalization. While mass deviations or differences in retention time face analytical variances, signal intensities of analytes depend on both analytical variances, such as signal suppression, and pre-analytical variances, such as dilution of a urine samples by different hydration states of donors. Therefore, normalization has to compensate for deviations in all three dimensions of data, the two defining parameters, e.g. retention time and molecular weight, in addition to signal intensity. Adequate approaches have been developed using either external standards or polypeptides that are found with high frequency in the analyzed samples and that serve as internal standards [24].

Statistical data mining when dealing with biological samples is often related to the special situation that the number of variables (polypeptides) highly outnumbers that of available samples. This generally results in a higher dimension of mathematical problems, and the selection of significant items has to consider respective adjustments for multiple hypothesis testing. Particularly, global procedures or adjustment of the $\mathrm{p}$ values, e.g. using classical Bonferroni correction, should be mandatory.

\section{Bioinformatic Approaches in Proteomics}

Medical definitions of diseases are often generic descriptions of observed symptoms and less is known about the exact pathological processes involved. Consequently, the concept of a single biomarker, allowing not only reliable diagnosis but also staging and prognosis, appears questionable. Combining multiple independent biomarkers to a diagnostic or predictive pattern may be adequate to address this problem. Many algorithms have emerged utilizing the available information on multiple biomarkers [28] with hierarchical decision tree-based classification methods [29], support vector machines [30], or Gaussian process [31] among them. A detailed discussion of the advantages and disadvantages of certain classification algorithms is beyond the scope of this review [for more detailed information, see 28]. Generally, every approach applied to a given classification problem should consider keeping the number of independent variables minimal and certainly below the number of datasets investigated. Under such circumstances, over-fitting or 
'memorizing' to the training set and thus poor classification of blinded datasets can be minimized. Independent of the algorithm applied, the generated classifiers should be imperatively validated using a blinded dataset in any report on potential biomarkers to exclude any memorizing and to quantify diagnostic performance [7].

\section{Aging}

The former sections tried to give a concise overview about the technical aspects of proteomics. The following sections will describe the application of these methods to questions on age-related chronic diseases. In this review, we will present some examples demonstrating general principles or potential future developments in the field of clinical proteomics, regarding diseases related to aging. We will start with the process of aging itself and go on with widespread diseases, such as diabetes, CAD, and different types of cancers (fig. 3).

Aging can be understood as a multidimensional process in an organism of physical, psychological, and social change over time. Some dimensions of aging grow and expand over time (e.g. knowledge of world events or experience), while others decline, such as proliferative capacity of cells or glomerular filtration rate of the kidney. Since the very first clinical observation of kidney diseases, it has been suspected that the urinary protein content can reflect the pathological changes in the kidney. Therefore, detailed analysis of the urinary proteome might also be informative on the physiological changes in the aging kidney. However, detailed high resolution analysis of urinary proteins and peptides has only recently become possible. We analyzed the low molecular weight urinary proteome of 324 healthy individuals from 2 to 73 years of age to gain insight into renal aging in humans. We observed age-related modification of expression in 325 of 5,000 urinary peptides. The majority of these changes was associated with renal development before and during puberty, while 49 peptides were related to aging in adults. Of these 49 peptides, the majority was also a marker of chronic kidney disease, suggesting high similarity between aging and chronic renal disease. The identification of a number of these age-related peptides as urinary fragments of collagen $\alpha 1$ type I suggests that modified proteolytic activity is involved in human renal ageing. These observations are in line with the main histological observations that the decline in glomerular filtration rate in the aging population is accompanied by glomerular sclerosis and increases in interstitial tissue, i.e. expansion of the extracellular matrix (ECM). Under physiological conditions, the ECM is thought to be maintained by equilibrium of synthesis and degradation. We observed that age modifies the occurrence of a number of urinary fragments of collagen $\alpha 1$ type I representing the proteolytic activity in the kidney [28]. We propose that the decrease in urinary collagen type I fragments with increasing age reflects a decrease in the activity of the renal proteolytic enzymes that is accompanied by increased renal ECM accumulation in the elderly and a reduction in urinary collagen type I fragments. This work pinpoints the role of synthetic and degradative processes in modulating cell machinery and emphasizes metabolic impairment with age.

In humans, telomere shortening occurs in the vast majority of tissues during physiological aging and is accelerated in chronic diseases that increase the rate of cell turnover. Therefore, telomere dysfunction limits the proliferative capacity of human cells by activation of DNA damage responses, inducing senescence or apoptosis. Recently, CRAMP, stathmin, EF-1, and chitinase were identified as marker proteins that are secreted from telomeredysfunctional bone marrow cells of late generation telomerase knockout mice (G4mTerc-/-) [32]. The expression levels of these proteins increase in blood and in various tissues of aging G4mTerc-/- mice, but not in aging mice with long telomere reserves. Orthologs of these proteins are upregulated in late passage presenescent human fibroblasts and in early passage human cells in response to DNA-damaging $\gamma$-irradiation. In a blinded study, the expression levels of these marker proteins were validated to increase in blood plasma of aging humans and to show further increase in geriatric patients with aging-associated diseases. Moreover, there was a significant increase in the expression of these biomarkers in the blood plasma of patients with chronic diseases that are associated with increased rates of cell turnover and telomere shortening, such as cirrhosis and myelodysplastic syndromes. These results support the concept that telomere dysfunction and DNA damage are interconnected pathways that are activated during human aging and disease. Furthermore, both telomere dysfunction and DNA damage may generate subsequent errors in the fidelity of protein synthesis according to the popular 'error catastrophe' theory of aging [33], proposing age-accumulating damage and cellular morphology changes. Therefore, it is important to understand and detect changes in physiological function and structure that normally occur with aging, and to distinguish these changes from alterations that take place with disease. 


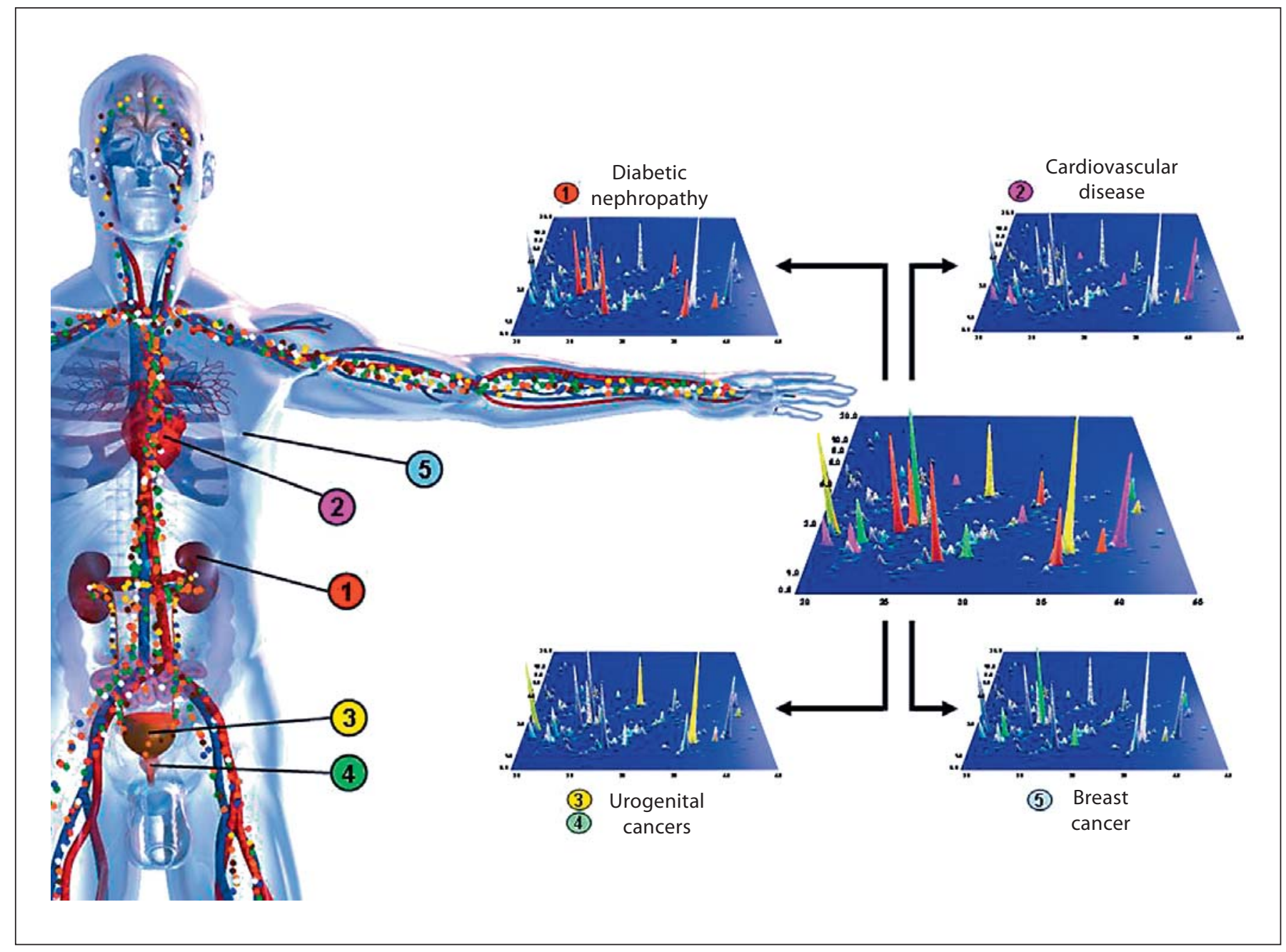

Fig. 3. Chronic age-related diseases. Age-related changes in the proteome have been described and were found to be highly similar to changes associated with chronic diseases. We give several examples on the successful application of proteomics in the diagnosis, prognosis and therapy of these chronic diseases. Longstanding diabetes is associated with a decline in renal function and diabetic nephropathy has become the most prevalent cause of end-stage renal disease (1). Patients with type 2 diabetes particularly progress to severe life-threatening coronary artery disease, the leading cause of morbidity and mortality worldwide (2). Can-

\section{Diabetic Nephropathy}

Long-standing diabetes is associated with a decline in renal function, and diabetic nephropathy has become the most prevalent cause of end-stage renal disease. Over the years, efforts have been made to find biomarkers early in the clinical course to better identify and treat individuals at high risk of diabetic nephropathy. cer can be considered a chronic disease predominately observed in the second half of life. Taken together, urological tumors of the prostate (3) and the bladder (4) are the most incident malignancies in the United States followed by breast cancer (5), and cause more than $35 \%$ of incidences. Essentially, all clinical proteomic investigations indicate that the approach to combine several disease-associated markers to a clearly distinct panel appears to be well suited for disease diagnosis, staging, progression, and for assessment of prognosis of these chronic diseases. 
Analysis of the urinary proteome in patients with type 2 diabetes mellitus using CE-MS revealed 168 urinary proteins present in over $90 \%$ of the samples, suggesting the existence of a consistent urinary proteome [35]. Several independent proteomic studies using 2DE or LC-MS support this hypothesis with the establishment of urinary proteome databases [36-38]. Biomarker patterns enabled not only the diagnosis of a specific (primary) kidney disease, but also the differential diagnosis with high sensitivity and specificity for the different kidney diseases, such as IgA nephropathy, focal-segmental glomerulosclerosis, membranous glomerulonephritis, minimalchange disease, and diabetic nephropathy. These findings have recently been validated in blinded, partially prospective clinical studies [39-41].

Interestingly, many of the identified discriminatory urinary polypeptides of patients with renal diseases are proteolytic fragments of larger proteins. These findings suggest that specific proteases in urine may cleave these excreted proteins as supported by a recent study of patients with nephrotic syndrome due to different glomerular diseases. The findings demonstrate specific urinary proteases that cleaved albumin and $\alpha 1$-antitrypsin. Almost 100 proteolytic fragments appeared as distinct spots on 2D gels [42].

Urinary proteome analysis may also be an excellent tool for fast, noninvasive, and unbiased monitoring of disease progression or response to therapy. In a randomized double-blinded placebo-controlled study, the treatment of macroalbuminuric patients was evaluated with daily doses of $8,16,32 \mathrm{mg}$ candesartan or placebo for 2 months [43]. Treatment with candesartan was significantly displayed in 15 of 113 proteins characteristic for diabetic nephropathy. Data from patients with vasculitis, where therapy improved or changed the vasculitis-specific protein pattern towards a normal urinary proteome, outline the potential of proteomic approaches in therapy monitoring [Haubitz et al., submitted; 42].

\section{Coronary Artery Disease}

Although CAD is a leading cause of morbidity and mortality worldwide and risk factors (dyslipidemia, diabetes, hypertension) and humoral markers of plaque vulnerability (C-reactive protein, interleukin-6, -10 and $-18, \mathrm{CD}-40 \mathrm{~L}$ ) have been identified, there is still little known about the underlying molecular causes. Certain subgroups of patients, such as patients with type 2 diabetes and the elderly, particularly progress to severe life- threatening CAD without many symptoms and signs [44].

Several groups have reported on the application of mainly $2 \mathrm{DE}$ proteomic techniques to analyze tissue or plaque specimens to study cardiovascular disease or arteriosclerosis. For instance, the protein expression profile of atherosclerotic tissue samples revealed 39 differentially expressed proteins [45]. These are involved in a number of biological processes, including calcium-mediated processes, migration of vascular smooth muscle cells, matrix metalloproteinase activation and regulation of proinflammatory cytokines. Confirmation of differential protein expression was performed by Western blot analysis. Additionally, the ferritin light chain was differentially expressed in 10 coronary arteries from patients with CAD compared to 7 vessels from healthy individuals. These results were subsequently confirmed by Western blot and quantitative real-time PCR analysis [46]. The authors concluded that increased expression of the ferritin light chain in diseased coronary arteries may be related to increased protein stability or upregulation of expression at the posttranscriptional level in the diseased tissues. Duran et al. [47] applied 2DE to carotid atherosclerotic plaques cultured in vitro. A comparison of the diseased vessel with normal controls revealed differential patterns of protein secretion [47].

Vaisar et al. [48] used LC-MS/MS shotgun proteomics to profile HDL composition. They compared isolates from healthy subjects and subjects with CAD and identified multiple complement-regulatory proteins and a pattern of serpins with serine-type endopeptidase inhibitor activity. Many acute-phase response proteins were also detected, supporting the proposal that HDL cholesterol is of central importance in inflammation [48].

All the above-mentioned studies did not yield novel diagnostic or prognostic biomarkers of CAD. Only a few studies focused on the analysis of body fluids derived through minimally invasive means. However, these studies were either based on pooled blood specimens [47, 48], making individual sample classification impossible, or they included only small patient cohorts without blinded studies for validation [48].

Recently, 370 urine samples from 88 patients with severe CAD and 282 controls were analyzed using CE-MS [49]. The ability of a CAD-specific pattern of 15 peptides to predict the presence of CAD was evaluated in a blinded study. The signature panel showed a sensitivity of $98 \%$ and a specificity of $83 \%$ in discriminating between the presence and absence of disease. Using peptide sequencing (top-down MS/MS approach), five of the polypep- 
tides constituting the $\mathrm{CAD}$-specific panel were identified as fragments of collagen $\alpha-1$ type I or III. These types of collagens are predominant proteins in the arterial walls and also appear together in the thickened intima of atherosclerotic lesions. The observation that all identified collagen fragments were upregulated in CAD samples compared to controls suggests that the elevated collagen degradation levels are related to increased collagenase activity. Interestingly, increased MMP-9 collagenase levels have been reported in patients with stable angiographic coronary atherosclerosis [50]. The established pattern demonstrated a response to exercise therapy. While predominantly inactive patients had no change in pattern. Physically active patients showed significant changes towards a 'healthier' biomarker pattern over 14 weeks, suggesting the possibility of measuring the effects of therapeutic interventions to assist personalized medicine strategies.

In a sub-cohort of the Coronary Artery Calcification in Type I Diabetes (CACTI) study, the same pattern was applied to urine samples from 19 individuals with no history of CAD at baseline and age-, sex- and diabetesmatched controls. The CAD score significantly predicted development of CAD (OR 2.2; 95\% CI 1.3-5.2; p = 0.0016) and remained significant when individually corrected for common CAD risk factors [Snell-Bergeon et al., in press]. These data strongly suggest that urinary proteome patterns can predict future cardiovascular events. Furthermore, this observation also provides a molecular basis for the link between the kidney and CAD, and may help to explain why chronic kidney disease is one of the best predictors for CAD [46].

In an independent study, Muhlen et al. [in press] analyzed urine and plasma samples of patients presenting with acute symptoms suspicious for CAD by CE-MS. All patients were subjected to coronary angiography and either assigned to a CAD or non-CAD group. Significant discriminatory power was achieved in urine, but not in plasma. The authors concluded that the reproducibility of proteomic plasma analysis may be limited by latent protease activity. In contrast, a combination of 17 urinary polypeptides allowed separation of both groups in a blinded study with an accuracy of $84 \%$. Sequencing of these discriminatory peptides identified fragments of collagen $\alpha 1$ (types I and III), which were furthermore demonstrated to be expressed in atherosclerotic plaques of the human aorta. These findings independently confirmed the observations of Zimmerli et al. [49].

\section{Malignancies}

Cancer can be considered as a chronic disease predominately observed in the second half of life. About 25\% of total deaths per year in the European Union are related to tumors. Taken together, prostate cancer $(\mathrm{PCa})$ and bladder cancer $(\mathrm{BCa})$ are the malignancies most often found in the United States followed by breast cancer. These three malignancies together caused more than 35\% of the cancer incidences in 2007 [http://www.cancer.org/ downloads/STT/EstimatedNewCancerCasesByState2007OldMethod.pdf], pinpointing the urgent need for novel biomarkers for cancer. In general, the applications of proteomic technologies for the discovery of cancer biomarkers are numerous, and a detailed overview is clearly beyond the possibilities of a single review. Therefore, we will focus on the three most incident malignancies and present examples demonstrating technological principles that already have proven clinical relevance.

\section{Prostate Cancer}

Since the advent of prostate-specific antigen (PSA) screening, a significant number of US men have had a PSA test. The screening led to a significant increase in the number of cases diagnosed [51]. Unfortunately, the specificity of PSA is around $70 \%$ and this results in up to 700,000 unnecessary prostate biopsies per year in the United States [52]. Additionally, it is impossible to distinguish clinically relevant $\mathrm{PCa}$ from cases requiring no further intervention. Therefore, if screening for this disease is to continue, then improved novel markers enabling this distinction are needed to avoid unnecessary biopsies and to recognize patients suitable for therapy.

It is no surprise that research attempts to integrate the potential benefits of novel technologies to address these current issues. SELDI profiling of tissue specimens has been used for the comparative analysis of laser capture microdissection-derived normal matched, high-grade prostatic intraepithelial neoplasia (PIN3) and malignant tumor cells [53]. In radical prostatectomy specimens, a dimeric form of mature growth factor 15 (GDF15) was found to be upregulated in PCa samples compared to PIN3 and normal controls. The quantitative proteomic profiling of tissue specimens using glycopeptide capturing followed by tandem MS showed tissue metalloproteinase inhibitor 1 to be downregulated in $\mathrm{PCa}$ [54].

Alternatively to the analysis of tissue specimens and their survey for diagnostic purposes, body fluids such as 
blood can be analyzed. SELDI-derived diagnostic patterns of proteins in human serum for PCa were first reported by Adam et al. [55]. They detected PCa patients with $83 \%$ sensitivity and $97 \%$ specificity in an independent validation set including 60 samples from patients with $\mathrm{PCa}$, benign prostatic hyperplasia (BPH) and healthy controls. Unfortunately, the results were subsequently heavily debated, predominantly because of the technological limitations of SELDI (see Proteomic Platforms section) and the instability of blood serum per se.

Therefore, the focus of proteomic profiling has somewhat shifted to urine to detect PCa. In first experiments, Rehman et al. [56] analyzed the urine of 6 patients suffering from $\mathrm{PCa}$ and $6 \mathrm{BPH}$ controls after prostate massage using 2DE mass fingerprinting. Urinary calgranulin B/MRP-14 was identified as a potential novel marker for PCa.

M'Koma et al. [57] introduced a method for discriminating $\mathrm{PCa}, \mathrm{PIN} 3$, and $\mathrm{BPH}$ by analyzing urine using reverse phase adsorption followed by MALDI-MS. Interestingly, the authors were able to discriminate PCa and BPH with lower diagnostic accuracy (67\% sensitivity and $71 \%$ specificity) than observed for PCa and HGPIN ( $81 \%$ sensitivity and $80 \%$ specificity), although the PIN3 state is histologically very similar to PCa.

Recently, the application of urinary profiling was described using CE online coupled to MS (CE-MS) in the noninvasive diagnosis of $\mathrm{PCa}$ [58]. In a blinded prospective study, a panel of 12 novel biomarkers for $\mathrm{PCa}(\mathrm{PCaP})$ was identified and validated. PCa-specific biomarkers could only be discovered in the first void urine (midstream urine samples did not enable identification of discriminatory molecules), suggesting that prostatic fluids may be the source of the defined biomarkers. Consequently, first void urine samples were tested for sufficient amounts of prostatic fluid, using a prostatic fluid indicative panel (IPP). A combination of IPP and PCaP to predict positive prostate biopsy was evaluated in the blinded prospective arm of the study. PCa was detected with $89 \%$ sensitivity and $51 \%$ specificity. Including age and percent free PSA in the proteomic signatures resulted in $91 \%$ sensitivity and $69 \%$ specificity.

This work underlines the importance of standardizing urine sampling for biomarker discovery. Thus, the questions whether to massage, the duration of the massage before collecting initial void, or whether to collect a standard void directly influence the ability to detect a given marker (panel) of prostate malignancy.

\section{Bladder Cancer}

Although the incidence of $\mathrm{BCa}$ is only a quarter of that observed for PCa, many patients suffering from bladder tumors show tumor recurrence, making $\mathrm{BCa}$ one of the most prevalent cancers worldwide. Therefore, especially screening for recurrence of $\mathrm{BCa}$ emphasizes the demand for reliable assays for the early detection of $\mathrm{BCa}$ recurrence once an initial tumor has been removed.

Using 2DE of urothelial tissue, BCa-specific nuclear matrix proteins (BCa antigen or BLCA-1, -4 , and -6 ) have been identified [59-62]. A sandwich immunoassay for BLCA-4 in urine discriminated patients with $\mathrm{BCa}$ with $95 \%$ specificity and $89 \%$ sensitivity [61]. Validation of these findings is currently underway in multicenter clinical trials.

Rasmussen et al. [63] extensively studied the urine of BCa patients using 2DE and could establish a database of 339 protein spots; 124 spots have been identified. In the following years, the same group successfully applied differential tissue profiling using $\left[{ }^{35} \mathrm{~S}\right]$-methionine labeling followed by 2DE-MS and Western blotting to define and validate novel biomarkers, such as $\gamma$-glutathione S-transferase, prostaglandin dehydrogenase, fatty acid-binding protein (FABP), and keratin for $\mathrm{BCa}$ diagnosis and tumor phenotyping [64]. In an independent study, FABP levels were assessed in 2,317 paraffin-embedded tissue blocks and were found to be associated with tumor grade and invasiveness [65]. Iwaki et al. [66] and Kageyama et al. [67] identified a panel consisting of calreticulin, $\gamma$-synuclein, and catechol-O-methyltransferase in $2 \mathrm{DE}$ profiling of tissue specimens and applied antibodies against the panel in Western blotting of urine. Based on $112 \mathrm{BCa}$ and 230 controls, sensitivity and specificity of 76.8 and $77.4 \%$ were obtained. However, a validation of these promising findings in a blinded prospective study is missing.

The diagnostic impact of tissue-derived markers remains directly related to the success of the tissue biopsy. Therefore, Vlahou et al. [68] described multiple transitional cell carcinoma (TCC)-associated protein changes in urine investigated using SELDI technology. The combination of these changes allowed BCa detection with a sensitivity of $87 \%$ and specificity of $66 \%$. One of the TCC biomarkers with a molecular weight of $3.4 \mathrm{kDa}$ was identified as $\alpha$-defensin. These proteins were also selected as TCC classifiers in a study by Munro et al. [14], which involved SELDI analysis of urine samples from a total of $227 \mathrm{BCa}$ patients and controls, and were also found to be overexpressed in invasive bladder tumors by immunohistochemistry [69]. The reported performance of SELDI 
profiles in detecting $\mathrm{BCa}$ in blinded sets of samples range from $72-93 \%$ sensitivity and $63-87 \%$ specificity $[14,15$, $68,70-73]$. Comparison of the datasets is not easy to achieve due to different chip surfaces and conditions used in the various studies.

Using CE-MS, Theodorescu et al. [5] profiled the urine samples of 46 patients with $\mathrm{BCa}$ and compared the obtained profiles with those of 33 healthy volunteers and 366 patients with malignant and non-malignant genitourinary diseases. A panel of 22 specific markers was validated in a blinded prospective study including $31 \mathrm{BCa}$ patients, 11 healthy individuals, and 138 patients with non-malignant genitourinary diseases with a sensitivity of $100 \%$ and specificity ranging from 86 (nephroliths) to $100 \%$ (healthy controls). Recently, the authors extended their approach to the noninvasive staging of $\mathrm{BCa}$, and validated a pattern of 4 urinary polypeptides discriminating superficial tumor stages (pTis, pTa and pT1) from muscle-invasive stages (pT2-4) with high accuracy [Schiffer et al., manuscript submitted].

\section{Breast Cancer}

Breast cancer is the most prevalent malignancy in women with good prognosis if diagnosed at an early stage. Systematic screening programs have increased the survival rate over the last decades. However, many patients still die from metastatic relapse. Breast cancer is a complex disease and many efforts have been made to study different types of breast tumors. The most frequent type is ductal breast cancer $(\sim 80 \%)$, while medullary breast cancer (MBC) is rarer. Morphological diagnosis remains difficult and little is known about features of aggressiveness, proliferation, and molecular alternations involved in the disease.

In the last decade, the application of proteomic methods to the analysis of breast cancer has primarily focused on tissue microarrays [for a more detailed review, see 74]. Using microarray technology, Jacquemier et al. [75] analyzed 18 proteins, including tumor suppressors, oncogenes, and proteins, on their clinical significance related to the proliferation and differentiation of breast tissue. They analyzed $61 \mathrm{MBC}$ and 300 grade 3 non-MBCs and characterized $\mathrm{MBCs}$ as being highly differentiated in basla/myoepithelial phenotype, defined by a logistic regression model including levels of P-cadherin, MIB1/ Ki67, ERBB2, and p53 using tissue microarrays. Using the same technique, the authors monitored expression of 26 selected proteins in more than 1,600 cancer samples from
552 consecutive patients with breast cancer in early stage [76]. The method identified a prognostic set of 21 proteins, significantly correlated to 5 -year metastasis-free survival, in a learning set of 368 patients $(\mathrm{p}<0.0001)$ and in a validation set of 184 patients $(\mathrm{p}<0.0001)$.

Moreira et al. [77] studied 14-3-3 $\sigma$ tumor suppressor as a key protein in breast cancer progression. They analyzed 65 primary breast carcinomas by immunostained $2 \mathrm{DE}$ and validated their findings in an independent set of 65 tumors using tissue microarrays. Surprisingly, downregulation of 14-3-3 $\sigma$ was sporadic in breast carcinomas, in contrast to the results found for $\mathrm{BCa}$ [78].

In order to define diagnostic biomarkers for breast cancer, SELDI analysis of blood as either serum or plasma was mainly applied [79-82] with sensitivities and specificities between $83-93$ and 87-93\%. However, only $\mathrm{Hu}$ et al. [82] validated their findings in an independent test set. The majority of these investigations suffer from limited patient numbers to sufficiently address the heterogeneity of the disease. Taking into account the methodological problems of the SELDI technology (see Proteomic Platforms section), these findings should be carefully reevaluated to substantiate the hypothesis of serum/plasma biomarkers of breast cancer.

Nipple aspirate fluid is an alternative body fluid more directly related to the tumor itself. Therefore, it might be seen as a potential source of breast cancer-specific diagnostic markers. There are some promising reports on the evaluation of the fluid and establishing methods to exploit the potential diagnostic information [83-85].

Recently, saliva was analyzed for breast cancer tumor markers using LC-MS/MS [86]. Although this body fluid is easily accessible and therefore an attractive source of biomarkers, the preliminary data have to be thoroughly validated in the future.

\section{Conclusions and Outlook}

A boost in disease-related proteomic information is expected in the very near future, which will also result in its broad clinical application. Compared to the high expectations generated by the more than 25 years of technological progress, the impact of proteomics on our understanding of the pathogenesis, diagnosis and treatment of diseases is modest, but significant. Essentially, all clinical proteomic investigations indicate that the approach to combine several disease-associated markers to a clearly distinct panel appears to be well suited for disease diagnosis, staging, progression, and the assessment of prog- 
nosis. However, a thorough validation of these signatures in well-designed blinded studies is essential and should therefore be mandatory [7].

To date, proteomics is still far from evolving its full potential as a tool in routine clinical settings. Nevertheless, there are several studies available including a substantial number of patients that clearly demonstrate its potential $[5,41,49,71]$. However, to take full advantage of the techniques, further standardization is needed to enable meta-analysis of datasets from different laboratories and a more fundamental knowledge in the context of clinically important questions. The Human Urine and Kidney Proteome Initiative within HUPO (http://hkupp. kir.jp) certainly provides invaluable help to this end.

\section{References}

1 Ginsburg GS, Haga SB: Translating genomic biomarkers into clinically useful diagnostics. Expert Rev Mol Diagn 2006;6:179-191.

$\checkmark 2$ Lindon JC, Holmes E, Nicholson JK: Metabonomics and its role in drug development and disease diagnosis. Expert Rev Mol Diagn 2004;4:189-199.

- 3 Omenn GS, States DJ, Adamski M, Blackwell TW, Menon R, Hermjakob H, Apweiler R, Haab BB, Simpson RJ, Eddes JS, Kapp EA, Moritz RL, Chan DW, Rai AJ, Admon A, Aebersold R, Eng J, Hancock WS, Hefta SA, Meyer H, Paik YK, Yoo JS, Ping P, Pounds J, Adkins J, Qian X, Wang R, Wasinger V, Wu CY, Zhao X, Zeng R, Archakov A, Tsugita A, Beer I, Pandey A, Pisano M, Andrews P, Tammen H, Speicher DW, Hanash SM: Overview of the HUPO Plasma Proteome Project: results from the pilot phase with 35 collaborating laboratories and multiple analytical groups, generating a core dataset of 3,020 proteins and a publicly-available database. Proteomics 2005;5:3226-3245.

4 Schaub S, Wilkins J, Weiler T, Sangster K, Rush D, Nickerson P: Urine protein profiling with surface-enhanced laser-desorption/ionization time-of-flight mass spectrometry. Kidney Int 2004;65:323-332.

5 Theodorescu D, Wittke S, Ross MM, Walden M, Conaway M, Just I, Mischak H, Frierson HF: Discovery and validation of new protein biomarkers for urothelial cancer: a prospective analysis. Lancet Oncol 2006;7:230-240.

-6 Fiedler GM, Baumann S, Leichtle A, Oltmann A, Kase J, Thiery J, Ceglarek U: Standardized peptidome profiling of human urine by magnetic bead separation and matrix-assisted laser desorption/ionization time-of-flight mass spectrometry. Clin Chem 2007;53:421-428.

-7 Mischak H, Apweiler R, Banks RE, Conaway M, Coon JJ, Dominizak A, Ehrich JH, Fliser D, Girolami M, Hermjakob H, Hochstrasser DF, Jankowski V, Julian BA, Kolch W, Massy Z, Neususs C, Novak J, Peter K, Rossing K, Schanstra JP, Semmes OJ, Theodorescu D, Thongboonkerd V, Weissinger EM, Van Eyk JE, Yamamoto T: Clinical proteomics: a need to define the field and to begin to set adequate standards. Proteomics Clin Appl 2007; 1:148-156.
-8 Kolch W, Neususs C, Pelzing M, Mischak H: Capillary electrophoresis-mass spectrometry as a powerful tool in clinical diagnosis and biomarker discovery. Mass Spectrom Rev 2005;24:959-977.

-9 Zürbig P, Renfrow MB, Schiffer E, Novak J, Walden M, Wittke S, Just I, Pelzing M, Neususs C, Theodorescu D, Root C, Ross M, Mischak H: Biomarker discovery by CE-MS enables sequence analysis via MS/MS with platform-independent separation. Electrophoresis 2006;27:2111-2125.

10 Annesley TM: Ion suppression in mass spectrometry. Clin Chem 2003;49:1041-1044.

11 Patterson SD, Aebersold R: Mass spectrometric approaches for the identification of gel-separated proteins. Electrophoresis 1995; 16:1791-1814

$12 \mathrm{Wu}$ TL: Two-dimensional difference gel electrophoresis. Methods Mol Biol 2006;328: 71-95.

13 Issaq HJ: The role of separation science in proteomics research. Electrophoresis 2001; 22:3629-3638.

14 Munro NP, Cairns DA, Clarke P, Rogers M, Stanley AJ, Barrett JH, Harnden P, Thompson D, Eardley I, Banks RE, Knowles MA: Urinary biomarker profiling in transitional cell carcinoma. Int J Cancer 2006;119:26422650

15 Zerefos P, Prados J, Kossida S, Kalousis A, Vlahou A: Sample preparation and bioinformatics in MALDI profiling of urinary proteins. J Chromatogr B Analyt Technol Biomed Life Sci 2007;853:20-30.

16 Najam-ul-Haq M, Rainer M, Trojer L, Feuerstein I, Vallant RM, Huck CW, Bakry R, Bonn GK: Alternative profiling platform based on MELDI and its applicability in clinical proteomics. Expert Rev Proteomics 2007;4:447-452.

17 Qian WJ, Jacobs JM, Liu T, Camp DG, Smith RD: Advances and challenges in liquid chromatography-mass spectrometry-based proteomics profiling for clinical applications. Mol Cell Proteomics 2006;5:1727-1744.
18 Kislinger T, Gramolini AO, Maclennan DH, Emili A: Multidimensional protein identification technology (MudPIT): technical overview of a profiling method optimized for the comprehensive proteomic investigation of normal and diseased heart tissue. J Am Soc Mass Spectrom 2005;16:1207-1220.

19 Soldi M, Sarto C, Valsecchi C, Magni F, Proserpio V, Ticozzi D, Mocarelli P: Proteome profile of human urine with two-dimensional liquid phase fractionation. Proteomics 2005;5:2641-2647.

-20 Bakry R, Huck CW, Najam-ul-Haq M, Rainer M, Bonn GK: Recent advances in capillary electrophoresis for biomarker discovery. J Sep Sci 2007;30:192-201.

21 Wu R, Hu L, Wang F, Ye M, Zou H: Recent development of monolithic stationary phases with emphasis on microscale chromatographic separation. J Chromatogr A 2008; 1184:369-392.

22 Schmitt-Kopplin P, Frommberger M: Capillary electrophoresis-mass spectrometry: 15 years of developments and applications. Electrophoresis 2003;24:3837-3867.

23 Hernandez-Borges J, Neususs C, Cifuentes A, Pelzing M: On-line capillary electrophoresis-mass spectrometry for the analysis of biomolecules. Electrophoresis 2004;25: 2257-2281.

24 Theodorescu D, Fliser D, Wittke S, Mischak $\mathrm{H}$, Krebs R, Walden M, Ross M, Eltze E, Bettendorf $\mathrm{O}$, Wulfing $\mathrm{C}$, Semjonow A: Pilot study of capillary electrophoresis coupled to mass spectrometry as a tool to define potential prostate cancer biomarkers in urine. Electrophoresis 2005;26:2797-2808.

25 Kozarova A, Petrinac S, Ali A, Hudson JW: Array of informatics: applications in modern research. J Proteome Res 2006;5:1051-1059.

-26 Shaw J, Rowlinson R, Nickson J, Stone T, Sweet A, Williams K, Tonge R: Evaluation of saturation labelling two-dimensional difference gel electrophoresis fluorescent dyes. Proteomics 2003;3:1181-1195.

-27 Wang W, Zhou H, Lin H, Roy S, Shaler TA, Hill LR, Norton S, Kumar P, Anderle M, Becker $\mathrm{CH}$ : Quantification of proteins and metabolites by mass spectrometry without isotopic labeling or spiked standards. Anal Chem 2003;75:4818-4826. 
28 Girolami M, Mischak H, Krebs R: Analysis of complex, multidimensional datasets. Drug Discov Today 2007;3:12-19.

29 Girolami M, Rogers S: Variational Bayesian multinomial probit regression with Gaussian process priors. Neural Comput 2006;18: 1790-1817.

30 Burges CJC: A tutorial on support vector machines for pattern recognition. Knowledge Discovery Data Mining 1998;2:121-167.

31 Rasmussen CE, Williams CKI: Gaussian Processes for Machine Learning. Boston, MIT Press, 2006

- 32 Jiang H, Schiffer E, Song Z, Wang J, Zürbig P, Thedieck K, Moes S, Bantel H, Saal N, Jantos J, Brecht M, Jeno P, Hall MN, Hager K, Manns MP, Hecker H, Ganser A, Dohner K, Bartke A, Meissner C, Mischak H, Ju Z, Rudolph KL: Proteins induced by telomere dysfunction and DNA damage represent biomarkers of human aging and disease. Proc Natl Acad Sci USA 2008;105:11299-11304.

-33 Orgel LE: Ageing of clones of mammalian cells. Nature 1973;243:441-445.

-34 Sharma K, Lee S, Han S, Lee S, Francos B, McCue P, Wassell R, Shaw MA, Ramachandra Rao SP: Two-dimensional fluorescence difference gel electrophoresis analysis of the urine proteome in human diabetic nephropathy. Proteomics 2005;5:2648-2655.

- 35 Mischak H, Kaiser T, Walden M, Hillmann M, Wittke S, Herrmann A, Knueppel S, Haller H, Fliser D: Proteomic analysis for the assessment of diabetic renal damage in humans. Clin Sci (Lond) 2004;107:485-495.

- 36 Thongboonkerd V, McLeish KR, Arthur JM, Klein JB: Proteomic analysis of normal human urinary proteins isolated by acetone precipitation or ultracentrifugation. Kidney Int 2002;62:1461-1469.

- 37 Adachi J, Kumar C, Zhang Y, Olsen JV, Mann M: The human urinary proteome contains more than 1,500 proteins including a large proportion of membranes proteins. Genome Biol 2006;7:R80.

- 38 Coon JJ, Zürbig P, Dakna M, Dominiczak AF, Decramer S, Fliser D, Frommberger M, Golovko I, Good DM, Herget-Rosenthal S, Jankowski J, Julian BA, Kellmann M, Kolch W, Massy Z, Novak J, Rossing K, Schanstra JP, Schiffer E, Theodorescu D, Vanholder R, Weissinger EM, Mischak H, Schmitt-Kopplin P: CE-MS analysis of the human urinary proteome for biomarker discovery and disease diagnostics. Proteomics Clin Appl 2008 2:964-973.

39 Haubitz M, Fliser D, Rupprecht H, Floege J, Haller $\mathrm{H}$, Rossing $\mathrm{K}$, Walden $\mathrm{M}$, Wittke $\mathrm{S}$, Mischak H: Defining renal diseases based on proteome analysis. Nephrol Dial Transplant 2005;20:V20.
40 Julian BA, Wittke S, Novak J, Good DM, Coon JJ, Kellmann M, Zürbig P, Schiffer E, Haubitz M, Moldoveanu Z, Calcatera SM, Wyatt RJ, Sykora J, Sladkova E, Hes O, Mischak H, McGuire BM: Electrophoretic methods for analysis of urinary polypeptides in IgA-associated renal diseases. Electrophoresis 2007;28:4469-4483.

41 Rossing K, Mischak H, Dakna M, Zürbig P, Novak J, Julian BA, Good DM, Coon JJ, Tarnow L, Rossing P: Proteomic discovery and validation of urinary biomarkers for diabetes and chronic renal disease. J Am Soc Nephrol 2008; in press.

42 Rossing K, Mischak H, Parving HH, Christensen PK, Walden M, Hillmann M, Kaiser $\mathrm{T}$ : Impact of diabetic nephropathy and angiotensin II receptor blockade on urinary polypeptide patterns. Kidney Int 2005;68: 193-205.

43 Rossing K, Christensen PK, Hansen BV, Carstensen B, Parving HH: Optimal dose of candesartan for renoprotection in type $2 \mathrm{di}$ abetic patients with nephropathy: a doubleblind randomized cross-over study. Diabetes Care 2003;26:150-155.

44 Verhave JC, Hillege HL, Burgerhof JG, Gansevoort RT, de Zeeuw D, de Jong PE; PREVEND Study Group: The association between atherosclerotic risk factors and renal function in the general population. Kidney Int 2005;67:1967-1973.

-45 Sung HJ, Ryang YS, Jang SW, Lee CW, Han $\mathrm{KH}, \mathrm{Ko}$ J: Proteomic analysis of differential protein expression in atherosclerosis. Biomarkers 2006;11:279-290.

46 You SA, Archacki SR, Angheloiu G, Moravec CS, Rao S, Kinter M, Topol EJ, Wang Q: Proteomic approach to coronary atherosclerosis shows ferritin light chain as a significant marker: evidence consistent with iron hypothesis in atherosclerosis. Physiol Genomics 2003; 13:25-30.

47 Duran MC, Martin-Ventura JL, Mas S, Barderas MG, Darde VM, Jensen ON, Egido J, Vivanco F: Characterization of the human atheroma plaque secretome by proteomic analysis. Methods Mol Biol 2007;357:141150.

48 Vaisar T, Pennathur S, Green PS, Gharib SA, Hoofnagle AN, Cheung MC, Byun J, Vuletic S, Kassim S, Singh P, Chea H, Knopp RH, Brunzell J, Geary R, Chait A, Zhao XQ, Elkon K, Marcovina S, Ridker P, Oram JF, Heinecke JW: Shotgun proteomics implicates protease inhibition and complement activation in the antiinflammatory properties of HDL. J Clin Invest 2007;117:746-756.

49 Zimmerli LU, Schiffer E, Zürbig P, Kellmann M, Mouls L, Pitt A, Coon JJ, Schmiederer RE, Mischak H, Peter K, Kolch W, Delles C, Dominiczak AF: Urinary proteomics biomarkers in coronary artery disease. Mol Cell Proteomics 2008;7:290-298.
50 Berhane BT, Zong C, Liem DA, Huang A, Le $\mathrm{S}$, Edmondson RD, Jones RC, Qiao X, Whitelegge JP, Ping P, Vondriska TM: Cardiovascular-related proteins identified in human plasma by the HUPO Plasma Proteome Project pilot phase. Proteomics 2005; 5:3520-3530.

51 Jemal A, Siegel R, Ward E, Murray T, Xu J, Smigal C, Thun MJ: Cancer statistics, 2006. CA Cancer J Clin 2006;56:106-130.

52 Presti JC Jr: Prostate cancer: assessment of risk using digital rectal examination, tumor grade, prostate-specific antigen, and systematic biopsy. Radiol Clin North Am 2000;38: 49-58.

53 Cheung PK, Woolcock B, Adomat H, Sutcliffe M, Bainbridge TC, Jones EC, Webber D, Kinahan T, Sadar M, Gleave ME, Vielkind J: Protein profiling of microdissected prostate tissue links growth differentiation factor 15 to prostate carcinogenesis. Cancer Res 2004;64:5929-5933.

-54 Liu AY, Zhang H, Sorensen CM, Diamond DL: Analysis of prostate cancer by proteomics using tissue specimens. J Urol 2005; 173:73-78

55 Adam BL, Qu Y, Davis JW, Ward MD, Clements MA, Cazares LH, Semmes OJ, Schellhammer PF, Yasui Y, Feng Z, Wright GL Jr: Serum protein fingerprinting coupled with a pattern-matching algorithm distinguishes prostate cancer from benign prostate hyperplasia and healthy men. Cancer Res 2002;62: 3609-3614.

56 Rehman I, Azzouzi AR, Catto JW, Allen S, Cross SS, Feeley K, Meuth M, Hamdy FC: Proteomic analysis of voided urine after prostatic massage from patients with prostate cancer: a pilot study. Urology 2004;64: $1238-1243$.

57 M’koma AE, Blum DL, Norris JL, Koyama T, Billheimer D, Motley S, Ghiassi M, Ferdowsi N, Bhowmick I, Chang SS, Fowke JH, Caprioli RM, Bhowmick NA: Detection of preneoplastic and neoplastic prostate disease by MADI profiling of urine. Biochem Biophys Res Commun 2006;353:829-834.

- 58 Wittke S, Schiffer E, Bauer HW: Kapillarelektrophorese gekoppelte Massenspektrometrie zur Proteomanalyse als innovative diagnostische Methode bei Prostata- und Blasenkrebs. Urologe A 2007;46:733-739.

59 Getzenberg RH, Konety BR, Oeler TA, Quigley MM, Hakam A, Becich MJ, Bahnson RR: Bladder cancer-associated nuclear matrix proteins. Cancer Res 1996;56:1690-1694.

60 Konety BR, Nguyen TS, Brenes G, Sholder A, Lewis N, Bastacky S, Potter DM, Getzenberg RH: Clinical usefulness of the novel marker BLCA- 4 for the detection of bladder cancer. J Urol 2000;164:634-639. 
61 Nielsen ME, Gonzalgo ML, Schoenberg MP, Getzenberg RH: Toward critical evaluation of the role(s) of molecular biomarkers in the management of bladder cancer. World J Urol 2006;24:499-508.

62 Van Le TS, Miller R, Barder T, Babjuk M, Potter DM, Getzenberg RH: Highly specific urine-based marker of bladder cancer. Urology 2005;66:1256-1260.

63 Rasmussen HH, Orntoft TF, Wolf H, Celis JE: Towards a comprehensive database of proteins from the urine of patients with bladder cancer. J Urol 1996;155:2113-2119.

- 64 Celis JE, Ostergaard M, Basse B, Celis A, Lauridsen JB, Ratz GP, Andersen I, Hein B, Wolf H, Orntoft TF, Rasmussen HH: Loss of adipocyte-type fatty acid binding protein and other protein biomarkers is associated with progression of human bladder transitional cell carcinomas. Cancer Res 1996;56: 4782-4790.

-65 Ohlsson G, Moreira JM, Gromov P, Sauter G, Celis JE: Loss of expression of the adipocytetype fatty acid-binding protein (A-FABP) is associated with progression of human urothelial carcinomas. Mol Cell Proteomics 2005;4:570-581.

-66 Iwaki H, Kageyama S, Isono T, Wakabayashi Y, Okada Y, Yoshimura K, Terai A, Arai Y, Iwamura $\mathrm{H}$, Kawakita M, Yoshiki T: Diagnostic potential in bladder cancer of a panel of tumor markers (calreticulin, gammasynuclein, and catechol-o-methyltransferase) identified by proteomic analysis. Cancer Sci 2004;95:955-961.

67 Kageyama S, Isono T, Iwaki H, Wakabayashi Y, Okada Y, Kontani K, Yoshimura K, Terai A, Arai Y, Yoshiki T: Identification by proteomic analysis of calreticulin as a marker for bladder cancer and evaluation of the diagnostic accuracy of its detection in urine. Clin Chem 2004;50:857-866.

68 Vlahou A, Schellhammer PF, Mendrinos S, Patel K, Kondylis FI, Gong L, Nasim S, Wright JG Jr: Development of a novel proteomic approach for the detection of transitional cell carcinoma of the bladder in urine. Am J Pathol 2001;158:1491-1502.
69 Holterman DA, Diaz JI, Blackmore PF, Davis JW, Schellhammer PF, Semmes OJ, Vlahou A: Overexpression of alpha-defensin is associated with bladder cancer invasiveness. Urol Oncol 2006;24:97-108.

70 Mueller J, von Eggeling F, Driesch D, Schubert J, Melle C, Junker K: ProteinChip technology reveals distinctive protein expression profiles in the urine of bladder cancer patients. Eur Urol 2005;47:885-893.

71 Vlahou A, Giannopoulos A, Gregory BW, Manousakas T, Kondylis FI, Wilson LL, Schellhammer PF, Wright GL Jr, Semmes OJ: Protein profiling in urine for the diagnosis of bladder cancer. Clin Chem 2004;50:14381441.

72 Zhang YF, Wu DL, Guan M, Liu WW, Wu Z, Chen YM, Zhang WZ, Lu Y: Tree analysis of mass spectral urine profiles discriminates transitional cell carcinoma of the bladder from noncancer patient. Clin Biochem 2004; 37:772-779.

73 Liu W, Guan M, Wu D, Zhang Y, Wu Z, Xu $\mathrm{M}, \mathrm{Lu} \mathrm{Y}$ : Using tree analysis pattern and SELDI-TOF-MS to discriminate transitional cell carcinoma of the bladder cancer from noncancer patients. Eur Urol 2005;47:456462.

74 Bertucci F, Birnbaum D, Goncalves A: Proteomics of breast cancer: principles and potential clinical applications. Mol Cell Proteomics 2006;5:1772-1786.

75 Jacquemier J, Padovani L, Rabayrol L, Lakhani SR, Penault-Llorca F, Denoux Y, Fiche M, Figueiro P, Maisongrosse V, Ledoussal V, Martinez PJ, Udvarhely N, El MG, Ginestier C, Geneix J, Charafe-Jauffret E, Xerri L, Eisinger F, Birnbaum D, Sobol H: Typical medullary breast carcinomas have a basal/myoepithelial phenotype. J Pathol 2005;207:260-268.

76 Jacquemier J, Ginestier C, Rougemont J, Bardou VJ, Charafe-Jauffret E, Geneix J, Adelaide J, Koki A, Houvenaeghel G, Hassoun J, Maraninchi D, Viens P, Birnbaum D, Bertucci F: Protein expression profiling identifies subclasses of breast cancer and predicts prognosis. Cancer Res 2005;65:767-779.

-77 Moreira JM, Ohlsson G, Rank FE, Celis JE: Down-regulation of the tumor suppressor protein 14-3-3sigma is a sporadic event in cancer of the breast. Mol Cell Proteomics 2005;4:555-569.
78 Moreira JM, Gromov P, Celis JE: Expression of the tumor suppressor protein 14-3-3 sigma is down-regulated in invasive transitional cell carcinomas of the urinary bladder undergoing epithelial-to-mesenchymal transition. Mol Cell Proteomics 2004;3:410-419.

79 Li J, Zhang Z, Rosenzweig J, Wang YY, Chan DW: Proteomics and bioinformatics approaches for identification of serum biomarkers to detect breast cancer. Clin Chem 2002;48:1296-1304.

80 Vlahou A, Laronga C, Wilson L, Gregory B, Fournier K, McGaughey D, Perry RR, Wright GL Jr, Semmes OJ: A novel approach toward development of a rapid blood test for breast cancer. Clin Breast Cancer 2003;4:203-209.

81 Becker S, Cazares LH, Watson P, Lynch H, Semmes OJ, Drake RR, Laronga C: Surfacedenhanced laser desorption/ionization timeof-flight (SELDI-TOF) differentiation of serum protein profiles of BRCA-1 and sporadic breast cancer. Ann Surg Oncol 2004;11: 907-914.

$82 \mathrm{Hu}$ Y, Zhang S, Yu J, Liu J, Zheng S: SELDITOF-MS: the proteomics and bioinformatics approaches in the diagnosis of breast cancer. Breast 2005; 14:250-255.

83 Paweletz CP, Trock B, Pennanen M, Tsangaris T, Magnant C, Liotta LA, Petricoin EF, III: Proteomic patterns of nipple aspirate fluids obtained by SELDI-TOF: potential for new biomarkers to aid in the diagnosis of breast cancer. Dis Markers 2001;17:301-307.

84 Sauter ER, Zhu W, Fan XJ, Wassell RP, Chervoneva I, Du Bois GC: Proteomic analysis of nipple aspirate fluid to detect biologic markers of breast cancer. Br J Cancer 2002;86: $1440-1443$.

85 Noble J, Dua RS, Locke I, Eeles R, Gui GP Isacke CM: Proteomic analysis of nipple aspirate fluid throughout the menstrual cycle in healthy pre-menopausal women. Breast Cancer Res Treat 2007;104:191-196.

- 86 Streckfus CF, Mayorga-Wark O, Arreola D, Edwards C, Bigler L, Dubinsky WP: Breast cancer related proteins are present in saliva and are modulated secondary to ductal carcinoma in situ of the breast. Cancer Invest 2008;26:159-167. 